\title{
TWO REMARKS ON THE GROUP ALGEBRA OF A FINITE GROUP
}

\begin{abstract}
K. L. FIELDS ${ }^{1}$
ABstract. If $K \subseteq Q\left(\zeta_{m}\right), m$ least, we find the smallest $n$ such that $M_{n}(K)$ appears in $Q G$ for some finite group $G$ when $m$ is either a prime power or not exactly divisible by a prime to the first power. We also show that every group of even order possesses a nontrivial real valued character of Schur index 1 over the rationals.
\end{abstract}

1. It is well known that if $M_{n}(K)$, the algebra of $n \times n$ matrices over the field $K$, appears as a simple component in the rational group algebra $Q G$ of a finite group, then $K \subseteq Q\left(\zeta_{m}\right)$ for some $m$; conversely, given $K \subseteq Q\left(\zeta_{m}\right)$ for some $m, M_{n}(K)$ appears in some $Q G$ for some $n$. We ask: What is the least such $n$ ? We can answer when $K \subseteq Q\left(\zeta_{p}^{a}\right)$, i.e., when $m$ is a power of a prime:

Let $\boldsymbol{Q} \subseteq K \subseteq Q\left(\zeta_{p}^{a}\right)$, and assume that $a$ is the smallest exponent of $p$ which suffices. It is clear that if $M_{n}(K)$ occurs in $Q G$ then $G$ contains an element of order $p^{a}$. Now if $A^{m}=1, m$ least, for some $A \in M_{n}(K)$, then $\phi(m) \leqq n[K: Q]$ (since the dimension over $Q$ of every maximal subfield of $M_{n}(K)$ equals $\left.n[K: Q]\right)$. In particular, $n$ must be at least $\left[Q\left(\zeta_{p}^{a}\right): K\right]$. We claim that this value of $n$ suffices: for let $G_{0}$ $=\operatorname{gal}\left(Q\left(\zeta_{p^{a}}\right) / K\right)$ and $G=\langle x\rangle \times_{s d} G_{0}$ where $x^{p^{a}}=1$ and $G_{0}$ acts on $x$ in the same way as it acts on $\zeta_{p}$. Then $Q G$ contains as a simple component the crossed product $\left\langle Q\left(\zeta_{p}^{a}\right), G_{0}, 1\right\rangle \cong M_{n}(K)$ where $n=$ $\left[Q\left(\zeta_{p}^{a}\right): K\right]$.

By Corollary 3 of Brauer [2], this argument also shows that $n_{\text {minimal }}$ is $\left[Q\left(\zeta_{m}\right): K\right]$ whenever $m$ is not exactly divisible by any prime to the first power.

2. It is well known that every group of even order possesses a nontrivial real valued character. We extend this to the following:

THEOREM. Every group of even order possesses a nontrivial real valued absolutely irreducible character whose Schur index over the rationals is 1 , which generates a field of odd degree over the rationals, and is of odd degree.

Received by the editors January 5, 1971.

AMS 1969 subject classifications. Primary 2080.

Key words and phrases. Theorem of Burnside and Blichfeldt, Brauer-Speiser Theorem.

${ }^{1}$ Research supported by the National Science Foundation. 
Proof. $|G|=\sum m_{Q}(\chi)^{2}[Q(\chi): Q]\left[\chi(1) / m_{Q}(\chi)\right]^{2}+1$ where the summation is over the nontrivial, nonconjugate, absolutely irreducible characters of $G$. Since $|G|$ is even, $[Q(\chi): Q]$ must be odd for at least one $\chi$. By the Brauer-Speiser Theorem [1], $m_{Q}(\chi)$ is either 1 or 2 for all such $\chi$, and so there must exist a nontrivial character $\nu$ (in fact, an odd number of them) such that $[Q(\nu): Q]$ is odd, $m_{Q}(\nu)=1$, and $\nu(1)$ is odd.

\section{REFERENCES}

1. R. Brauer, Representations of finite groups, Lectures on Modern Math., vol. 1, Wiley, New York, 1963, pp. 133-175. MR 31 \#2314.

2. - A note on theorems of Burnside and Blichfeldt, Proc. Amer. Math. Soc. 15 (1964), 31-34. MR 28 \#1232.

University of Chicago, Chicago, Illinois 60637 